\title{
Efficacy and motor complications of original and generic levodopa in Parkinson's disease treatment
}

\author{
This article was published in the following Dove Press journal: \\ Neuropsychiatric Disease and Treatment \\ II May 2016 \\ Number of times this article has been viewed
}

\author{
Narongrit Kasemsap' \\ Satrirat Onsanit ${ }^{2}$ \\ Piyawan Chiewthanakul ${ }^{3}$ \\ Kannikar Kongbunkiat ${ }^{1}$ \\ Chonthicha Tanking' \\ Nisa Vorasoot' \\ Kittisak Sawanyawisuth ${ }^{1,4}$ \\ Somsak Tiamkao ${ }^{1,5}$ \\ 'Department of Medicine, Faculty \\ of Medicine, Khon Kaen University, \\ Khon Kaen, ${ }^{2}$ Department of Medicine, \\ Udonthani Hospital, Udonthani, \\ ${ }^{3}$ Department of Medicine, Khon Kaen \\ Hospital, ${ }^{4}$ Research Center in \\ Back, Neck, Other Joint Pain and \\ Human Performance (BNOJPH), \\ ${ }^{5}$ Neuroscience Research and \\ Development Group (NRDG), Khon \\ Kaen University, Khon Kaen, Thailand
}

Correspondence: Somsak Tiamkao Department of Medicine, Faculty of Medicine, Khon Kaen University, Khon Kaen 40002, Thailand

Tel +6643363664

Fax +66 43348399

Email somtia@kku.ac.th
Background: In general, a generic drug is considered interchangeable with the original formulated drug. In Parkinson's disease (PD), generic drug use remains debated. This study was aimed to investigate whether the generic drug was as effective as the original in improving the symptoms of PD and the prevalence of motor complications.

Methods: This study was a multicenter cohort study of patients with PD enrolled from three northeast hospitals in Thailand between February 2013 and February 2014. The patients were categorized into original and generic levodopa groups. The clinical characteristics, efficacy, and motor complications were compared between the groups.

Results: There were 400 eligible patients. Of these, 327 patients $(81.75 \%)$ met the study criteria and were classified as the original levodopa group (200 patients, 61.16\%) and the generic levodopa group (127 patients, 38.84\%). The average age of all patients with PD was 65 years. The duration of PD and the modified Hoehn-Yahr stages were not different between the groups. The total doses of original and generic levodopa-equivalent doses were significantly different (199.97 \pm 127.08 versus $305.58 \pm 138.27 \mathrm{mg} ; P$-value $<0.001)$ and the actual doses were $198.10 \pm 117.92$ versus $308.85 \pm 139.40 \mathrm{mg}$ ( $P$-value $<0.001)$. Approximately $80 \%$ of patients with PD in both groups had good responses ( $P$-value $>0.999)$, but the development of motor complications was significantly greater in the original than in the generic group.

Conclusion: Generic levodopa was effective in improving the symptoms of PD. The prevalence of motor complications in the original compound group, at a lower dose of levodopa equivalent, was higher than in the generic group.

Keywords: Parkinson's disease, original, generic, levodopa, efficacy, motor complication

\section{Introduction}

Parkinson's disease (PD) is a progressive neurodegenerative disorder caused due to dopamine depletion. It is estimated that the prevalence of PD in Thailand is 424.57 per 100,000 population. ${ }^{1}$ PD is mainly a movement disorder with four typical cardinal symptoms, including bradykinesia, rigidity, tremor, and postural instability. ${ }^{2-4}$ The gold standard treatment of PD is dopamine replacement. ${ }^{5-8}$ Levodopa is the firstline treatment and is highly effective for improving the symptoms of PD. Long-term levodopa treatment may cause several motor complications such as dyskinesia or dystonia. ${ }^{9}$

Thailand and other developing countries have an issue with the price of the original medications including levodopa. The original levodopa or Madopar ${ }^{\circledR}$ has a higher price as compared to the generic levodopas, Vopar or Levomet. As a result, original levodopa is limited for clinical use and only available in university or tertiary care hospitals. Generic levodopa is widely used in community or general hospitals, but its efficacy compared with that of original is still of concern. Studies comparing original 
and generic compounds are very difficult to perform, as most patients at the university hospital are on the original compound while the community and general hospitals are mandated to use only the generic compound with a few exceptions. This study was aimed to investigate whether the generic levodopa was as effective as the original levodopa in improving the symptoms of PD. Also, the prevalence of motor complications between the original and generic levodopa was compared.

\section{Materials and methods Study design}

This study was a multicenter consecutive case cohort study of patients with PD enrolled from three northeast hospitals in Thailand, including Udonthani Hospital, Khon Kaen Hospital, and Srinagarind Hospital of Khon Kaen University. The first two hospitals are general hospitals. Patients who had been diagnosed with PD according to the UK PD Brain Bank Clinical Diagnostic Criteria and were treated by neurologists were studied. The study period was between February 2013 and February 2014. The study protocol was approved by the ethics committees for human research of all three hospitals.

\section{Subjects}

All eligible patients were treated by the attending physicians and were categorized by the type of levodopa given, original or generic. Baseline characteristics, modified Hoehn-Yahr (H-Y) stage, duration of disease, treatment, dosage of levodopa, and motor complications were recorded. The dose of levodopa could be adjusted, but the type of levodopa for each patient remained the same throughout the study period. The modified H-Y stages were used to classify the involvement of PD as follows: 1) unilateral involvement only; 1.5) unilateral and axial involvement; 2) bilateral involvement without impairment of balance; 2.5) mild bilateral disease without recovery on pull test; 3 ) mild to moderate bilateral disease, some postural instability but physically independent; 4) severe disability, still able to walk or stand unassisted; and 5) wheelchair bound or bedridden unless aided. The responses to treatments were classified for each patient using the ratings for subjective improvement as good or poor. The motor complications were classified as motor fluctuation and dyskinesia. Motor fluctuations are the variable and unpredictable benefits of treatment of parkinsonian features in response to a dose of levodopa. Dyskinesia is the levodopa-induced involuntary movement that commonly occurs at the time of peak concentration of the dose of levodopa. The time of peak concentration was from the start of levodopa use to the first report of a motor complication.
Levodopa is the mainstay of treatment for motor symptoms of PD. Levodopa therapy, however, is associated with motor complications, particularly at higher doses. Therefore, an alterative strategy is to use lower doses of levodopa to reduce the motor complications of the monotherapy and using a dopamine agonist or monoamine oxidase type $\mathrm{B}$ inhibitor or adjunct therapy combining a dopamine agonist, a monoamine oxidase type $\mathrm{B}$ inhibitor, or a catechol-O-methyltransferase inhibitor with a lower dose of levodopa. To facilitate the comparisons between each strategy of treatment, a levodopa equivalent dose (LED) was obtained by adding together all antiparkinsonian medications that the patient received. The total levodopa dose equivalents were calculated as follows: (regular levodopa dose $\times 1)+($ levodopa continuous-release dose $\times 0.75)+$ $($ pramipexole dose $\times 67)+($ ropinirole dose $\times 16.67)+($ pergolide dose $\times 100)+($ bromocriptine dose $\times 10)+$ regular levodopa dose $+($ continuous-release levodopa dose $\times 0.75)] \times 0.25$, if the patient is taking entacapone. ${ }^{10}$

\section{Sample size calculation}

Assuming that a $20 \%$ difference between original and generic levodopa treatment response is significant, the minimal number of subjects needed was 89 . This calculation was performed by using the compared hypothesis for calculating the least number needed for each group.

\section{Statistical analyses}

After providing written informed consent, all patients were categorized into two groups by the type of levodopa: original and generic. Numerical factors were compared by either Student's $t$-test or Wilcoxon rank-sum test where appropriate, whereas categorical factors were compared by using chi-square or the Fisher's exact test where appropriate. All statistical analyses were performed by the Statistical Package for the Social Sciences statistical package version 22.0 (IBM Corporation, Armonk, NY, USA) and STATA version 10.0 (StataCorp LP, College Station, TX, USA). Statistical significance was defined as $P$-value $<0.05$.

\section{Results}

There were 400 eligible patients: 200 patients from Srinagarind Hospital, 100 patients from Udonthani Hospital, and 100 patients from Khon Kaen Hospital. Of these, 327 patients $(81.75 \%)$ met the study criteria and were classified as original levodopa group (200 patients, 61.16\%) and generic levodopa group (127 patients, 38.84\%). Two brands of generic levodopa were used in this study: 51 (40.2\%) patients used Vopar and 76 (59.8\%) patients used Levomet. All the 
Table I Demographic and baseline characteristics

\begin{tabular}{|c|c|c|c|}
\hline Variables & Original $(n=200)$ & Generic $(n=127)$ & $P$-value \\
\hline Male, n (\%) & I I 4 (57.0) & $62(48.82)$ & 0.14 \\
\hline Age, years (mean $\pm S D)$ & $65.68 \pm 11.80$ & $65.11 \pm 9.98$ & 0.65 \\
\hline \multicolumn{4}{|l|}{ Health insurance } \\
\hline Civil Servant Medical Benefits System & $123(61.50)$ & $18(14.17)$ & $<0.001$ \\
\hline Universal coverage & $57(28.50)$ & $105(82.68)$ & \\
\hline Social security & $2(1.0)$ & $4(3.15)$ & \\
\hline Self-paid & $18(9.0)$ & $0(0)$ & \\
\hline \multicolumn{4}{|l|}{ Ethnicities } \\
\hline Thai & $190(95.0)$ & 120 (94.49) & 0.18 \\
\hline Chinese & $7(3.50)$ & $2(1.57)$ & \\
\hline Mixed Thai and Chinese & $2(1.0)$ & $5(3.94)$ & \\
\hline Laotian & I (0.50) & $0(0)$ & \\
\hline Smoking, $n(\%)(n=2 I I)$ & $36(40.45)$ & $43(35.25)$ & 0.44 \\
\hline Age of onset, years (mean $\pm S D)$ & $60.12 \pm 11.83$ & $59.86 \pm 10.43$ & 0.84 \\
\hline Disease duration, years (mean $\pm \mathrm{SD}$ ) & $5.59 \pm 4.52$ & $5.25 \pm 4.09$ & 0.60 \\
\hline Total levodopa dose, mg (mean \pm SD) & $199.97 \pm 127.08$ & $305.58 \pm 138.27$ & $<0.001$ \\
\hline Levodopa equivalent dose (mean $\pm \mathrm{SD})$ & $198.10 \pm 117.92$ & $308.85 \pm 139.40$ & $<0.001$ \\
\hline Modified Hoehn-Yahr stage, n (\%) & & & 0.07 \\
\hline I & $103(51.50)$ & $59(46.46)$ & \\
\hline 1.5 & $25(12.50)$ & $10(7.87)$ & \\
\hline 2 & $55(27.50)$ & $50(39.37)$ & \\
\hline 2.5 & $12(6.0)$ & $4(3.15)$ & \\
\hline 3 & $5(2.50)$ & $2(1.57)$ & \\
\hline 4 & $0(0)$ & $2(1.57)$ & \\
\hline 5 & 0 & 0 & \\
\hline
\end{tabular}

Abbreviation: SD, standard deviation.

patients used the same brands throughout the study. Seventythree patients were excluded because of not using levodopa (six patients) or changing use between original and generic (44 patients) or were lost to follow-up (23 patients).

The average age of all patients with PD treated with original and generic levodopa was 65 years and approximately half of the patients were male (Table 1). Other factors were comparable, except for the type of insurance (Table 1). Most patients treated with generic levodopa had universal coverage (105 patients, $82.68 \%$ ), while most patients who received original levodopa had the Civil Servant Medical Benefits System insurance (123 patients, $61.50 \%$ ). The duration of $\mathrm{PD}$ in the generic group was not different from the original group at $5.59 \pm 4.52$ versus $5.25 \pm 4.09$ years ( $P$-value 0.06 ). The H-Y stages were not different between the groups.

The total doses of original levodopa and generic LEDs were significantly different $(199.97 \pm 127.08$ versus $305.58 \pm 138.27 \mathrm{mg}[P$-value $<0.001])$, and the actual doses were $198.10 \pm 117.92$ versus $308.85 \pm 139.40 \mathrm{mg}$ ( $P$-value $<0.001)$.

Approximately $80 \%$ of patients with $\mathrm{PD}$ in both groups had good responses, as shown in Table $2(P$-value $>0.999)$, but the development of motor complications was significantly greater in the original than in the generic group as shown in Table 3. The times to develop motor fluctuations at $48.12 \pm 33.20$ versus $62.10 \pm 39.55$ months ( $P$-value 0.07 ) and dyskinesia at $62.46 \pm 37.72$ versus $83.52 \pm 52.74$ months ( $P$-value 0.13$)$ were not significantly different between the original and generic groups (Table 2 ). There were no reports of major adverse drug events in either group.

\section{Discussion}

Clinical features of PD in the present study were quite similar to those in a previous study on Thai population by Kulkantrakorn et al. ${ }^{11}$ Sex and average ages were nearly the same in the original formulation and generic groups.

Table 2 Response to levodopa and development of motor complications

\begin{tabular}{llll}
\hline Variables & Original $(\mathbf{n}=\mathbf{2 0 0})$ & Generic $(\mathbf{n}=\mathbf{I 2 7})$ & \\
\hline Levodopa response, $\mathrm{n}(\%)$ & & & \\
Good & $164(82.0)$ & $104(81.9)$ & $>0.999$ \\
Poor & $36(18.0)$ & $23(18.1)$ & $38(29.92)$ \\
Development of motor complication, $\mathrm{n}(\%)$ & $83(41.50)$ & 0.035 \\
\hline
\end{tabular}


Table 3 Motor complications and levodopa dosage comparing original and generic compounds

\begin{tabular}{|c|c|c|c|}
\hline Developed motor complications & Original $(n=83)$ & Generic $(n=38)$ & $P$-value \\
\hline Modified Hoehn-Yahr stage, n (\%) & & & 0.61 \\
\hline I & $52(62.65)$ & $21(55.26)$ & \\
\hline 1.5 & $10(12.05)$ & $3(7.89)$ & \\
\hline 2 & $18(21.69)$ & $13(34.21)$ & \\
\hline 2.5 & $2(2.4 \mathrm{I})$ & I (2.63) & \\
\hline 3 & $I(1.20)$ & $0(0)$ & \\
\hline 4 & $0(0)$ & $0(0)$ & \\
\hline 5 & $0(0)$ & $0(0)$ & \\
\hline Time of levodopa use to motor fluctuation, months (mean \pm SD) & $48.12 \pm 33.20$ & $62.10 \pm 39.55$ & 0.07 \\
\hline Time of levodopa use to dyskinesia, months (mean \pm SD) & $62.46 \pm 37.72$ & $83.52 \pm 52.74$ & 0.13 \\
\hline Total levodopa dose, mg (mean \pm SD) & $229.51 \pm 143.03$ & $365.81 \pm 154.09$ & $<0.001$ \\
\hline Levodopa equivalent dose, $\mathrm{mg}($ mean $\pm \mathrm{SD})$ & $225.53 \pm 138.02$ & $371.07 \pm 154.87$ & $<0.001$ \\
\hline
\end{tabular}

Abbreviation: SD, standard deviation.

The present study compared original and generic levodopa groups for symptomatic responses in order to determine if the generic form was as good as or inferior to the original levodopa (original group $82.0 \%$, generic group $81.9 \%$; $P$-value $>0.999)$. The total dosage of levodopa used in the original group was lower than in the generic group (original group $199.97 \mathrm{mg}$, generic group $305.58 \mathrm{mg}$; $P$-value $<0.001)$. The LED used in the original group was $198.10 \mathrm{mg}$ versus $308.85 \mathrm{mg}$ in the generic group $(P$-value $<0.001)$. The present study showed that the dosage of levodopa used in treatment of patients with PD who had developed motor complications in the generic group was higher than the dosage used in the original group by approximately 1.5 times. Gasser et a ${ }^{12}$ reported on the pharmaceutical quality of generic levodopa/ benserazide products compared with original Madopar/ Prolopa $^{\circledR}$ (Roche, Switzerland) and found deviations in the active ingredients, a marked excess in degradation, and some problems with the dissolution times of generic compounds. Therefore, the higher dose of generics may be due to lower efficacy of the generic levodopa than the original. The US Food and Drug Administration published draft guidelines on levodopa/carbidopa showing that the bioequivalent base is at $90 \%$ confidence interval $;{ }^{13}$ therefore, the dosage used in the generic group was higher than that in the original group of the cited study. To date, there has been no long-term study on the bioequivalence of levodopa/benserazide in patients with PD. Pahwa et $\mathrm{al}^{14}$ who compared single doses of sinemet and atamet (generic carbidopa/levodopa) in patients with PD found that there was no statistically significant difference between sinemet and atamet in the time taken to reach the maximum concentration $\left(\mathrm{T}_{\max }\right)$, the maximum concentration $\left(\mathrm{C}_{\max }\right)$, area under the curve, and the motor performance tests, but there has been no study comparing the original and generic forms in the long-term use of levodopa.
Patients with PD who often use multiple drugs and have low gastric motility may experience drug-drug interactions and slow absorption of orally administered compounds in the morning. ${ }^{15}$ Madopar, the original levodopa, was slightly more expensive than Vopar and Levopar, the generic levodopa (US\$ 0.32 and 0.23 per tablet, respectively). The generic drugs, however, are used at higher doses than the original drug (305.58 \pm 138.27 versus $199.97 \pm 127.08 \mathrm{mg})$; therefore, it may not be cost-effective to substitute generic drugs for the brand name product.

The present study showed that a number of patients developed complications similar to the previous study. ${ }^{11}$ The incidence of complications in the original group was $41.50 \%$ and in the generic group was $29.92 \%$. The original group reported more motor complications ( $P$-value 0.035 ), but used lower doses of total levodopa and LEDs than the generic group. Further study on the pharmacokinetics and pharmacodynamics of original and generic levodopa in patients with PD will need to be carried out before considering the use of generic levodopa as a cost-effective treatment. Even though this study was of cohorts and similar to real-life clinical practice, a further large randomized controlled trial should be performed to confirm the results of this study. The lower rate of motor complications in the generic group may be due to the lower efficacy on a dosage basis of the generics versus the original levodopa. The evaluation of motor complications by asking patients rather than critical clinical evaluation using the Unified PD Rating Scale as used in real clinical practice could result in underdiagnosis. This, in itself, is a possible limitation of this study.

\section{Conclusion}

Generic levodopa was effective in improving the symptoms of PD. The prevalence of motor complications in the original 
compound group, at a lower dose of levodopa equivalent, was higher than in the generic group.

\section{Acknowledgments}

We would like to thank Prof James A Will for editing the MS via Publication Clinic KKU, Thailand. This work was supported by the Thailand Research Fund (TRF). Medline Co., Ltd. Thailand supported the study with the research fund and had no role in the study design, data collection, data analysis, and in obtaining any permission to access all data in this study.

\section{Disclosure}

The authors report no conflicts of interest in this work.

\section{References}

1. Bhidayasiri R, Wannachai N, Limpabandhu S, et al. A national registry to determine the distribution and prevalence of Parkinson's disease in Thailand: implications of urbanization and pesticides as risk factors for Parkinson's disease. Neuroepidemiology. 2011;37:222-230.

2. Schapira AHV, Olanow CE. Principles of Treatment in Parkinson's Disease. Philadelphia, PA: Elsevier Health Sciences; 2005.

3. Fahn S, Sulzer D. Neurodegeneration and neuroprotection in Parkinson disease. NeuroRx. 2004;1(1):139-154.

4. Sulzer D. Multiple hit hypotheses for dopamine neuron loss in Parkinson's disease. Trends Neurosci. 2007;30(5):244-250.
5. Yahr MD, Duvoisin RC, Schear MJ, Barrett RE, Hoehn MM. Treatment of parkinsonism with levodopa. Arch Neurol. 1969;21(4):343-354.

6. Hauser R, Zesiewicz T. Advances in the pharmacologic management of early Parkinson disease. Neurologist. 2007;13(3):126-132.

7. Jankovic J, Stacy M. Medical management of levodopa-associated motor complications in patients with Parkinson's disease. CNS Drugs. 2007;21(8):677-692.

8. Esposito E, Cuzzocrea S. New therapeutic strategy for Parkinson's and Alzheimer's disease. Curr Med Chem. 2010;17(25):2764-2774.

9. Contin M, Martinelli P. Pharmacokinetics of levodopa. J Neurol. 2010;257(Suppl 2):S253-S261.

10. Hobson D, Lang A, Martin W, Razmy A, Rivest J, Fleming J. Excessive daytime sleepiness and sudden-onset sleep in Parkinson disease: a survey by the Canadian Movement Disorders Group. JAMA. 2002; 287(4):455-463.

11. Kulkantrakorn K, Tiamkao S, Pongchaiyakul C, Pulkes T. Levodopa induced motor complications in Thai Parkinson's disease patients. J Med Assoc Thai. 2006;89:632-637.

12. Gasser UE, Fischer A, Timmermans JP, Arnet I. Pharmaceutical quality of seven generic Levodopa/Benserazide products compared with original Madopar $^{\circledR} /$ Prolopa $^{\circledR}$. BMC Pharmacol Toxicol. 2013;14(1):24.

13. US Food and Drug Administration. Draft Guidance on Carbidopa; Levodopa. Recommended; September 2015. Available from: http:// www.fda.gov/downloads/Drugs/GuidanceComplianceRegulatory Information/Guidances/UCM460981.pdf. Accessed August 20, 2015.

14. Pahwa R, Marjama J, McGuire D, et al. Pharmacokinetic comparison of sinemet and atamet (generic carbidopa/levodopa): A single-dose study. Mov Disord. 1996;11:427-430.

15. Go C, Rosales R, Schmidt P, Lyons KE, Pahwa R, Okun MS. Generic versus branded pharmacotherapy in Parkinson's disease: does it matter? A review. Parkinsonism Relat Disord. 2011;17(5):308-312.
Neuropsychiatric Disease and Treatment

\section{Publish your work in this journal}

Neuropsychiatric Disease and Treatment is an international, peerreviewed journal of clinical therapeutics and pharmacology focusing on concise rapid reporting of clinical or pre-clinical studies on a range of neuropsychiatric and neurological disorders. This journal is indexed on PubMed Central, the 'PsycINFO' database and CAS,

\section{Dovepress}

and is the official journal of The International Neuropsychiatric Association (INA). The manuscript management system is completely online and includes a very quick and fair peer-review system, which is all easy to use. Visit http://www.dovepress.com/testimonials.php to read real quotes from published authors. 